\title{
DISTRIBUTIVE POSTULATES FOR SYSTEMS LIKE BOOLEAN ALGEBRAS
}

\author{
BY \\ GEORGE D. BIRKHOFF AND GARRETT BIRKHOFF
}

1. Introduction. Boole [2, p. 41] $\left.{ }^{1}\right)$ pointed out a close analogy between ordinary algebra and the "algebra of logic," now called Boolean algebra. Both have operations of addition and multiplication which are commutative and associative; both have a 0 for addition and multiplication and a 1 for multiplication; in both, multiplication is distributive on sums.

The connection was first made precise by Stone [5]. Stone defined a "Boolean ring" as a ring in which $a a=a$, and showed that this implies $a+a=0$ and $a b=b a$. He showed that by simple constructions, one could transform Boolean algebras into Boolean rings and vice versa.

Stone and most earlier authors (see Huntington [3]) used commutative and associative laws. In a remarkable paper [4], Newman based his developments entirely on distributivity, the existence of complements, and the properties of 0 and 1 . Every such distributive, complemented algebra is the direct sum of the Boolean subalgebra of elements satisfying $a+a=a$, and the (not necessarily associative) Boolean subring of elements satisfying $\left({ }^{2}\right) a+a=0$.

During lectures on Boolean algebra, but using stronger postulates, G. D. Birkhoff independently discovered Newman's decomposition theorem. Our discussion of this in the summer of 1944 led to the results presented below.

As Newman's postulates are independent (cf. J. London Math. Soc. vol. 17 (1942) pp. 34-47 and vol. 14 (1944) pp. 28-30), we have been unable to weaken them. However, we have been able to make large parts of his argument much shorter and simpler, at the cost of a weak additional postulate ( $\mathrm{P}^{\prime}$ ' below), added to those of his Theorem $1 \mathrm{~b}$. We show that one need only apply Boole's method of expansion [2, p. 151], systematically.

We first show (\$2) that the postulates are left-right symmetric by a new and simple argument. We then show $(\$ 3)$ that the existence and properties of "even" elements follow from more general considerations than those of Newman, and give $(\$ 4)$ a simplified proof of his decomposition theorem. In $\S 5$, we give a new set of postulates for distributive lattices; in $\S 6$, we give an

Presented to the Society, February 23, 1946; received by the editors December 5, 1945.

(1) Numbers in brackets refer to the Bibliography at the end of the paper.

(2) It is easily shown that any "idempotent" ring (in which $a a=a$ for all $a$ ) satisfies $a+a=0$ and $a b=b a$; the proof of [5] applies to the nonassociative case. Conversely, in any such ring, $a+1$ is a complement of $a$. Finally, any idempotent and commutative multiplication table for basis-elements defines an idempotent ring (cf. L. E. Dickson, Algebras and their arithmetics, Chicago, 1922, p. 22); the idempotence follows from the binomial theorem and commutativity. 
entirely novel approach to postulates for Boolean algebras and (associative) Boolean rings. Finally, in $\$ 7$, we show that in addition to the "metamathematical" principles of duality and left-right symmetry, only three postulates are needed for Boolean algebras and three for distributive lattices-but that two are not quite enough.

2. Postulates; first deductions. Consider any system with two binary operations which satisfies the following postulates:

P1. $a(b+c)=a b+a c$.

$\mathrm{P}^{\prime} .(a+b) c=a c+b c$.

P2. $\exists 1$, such that $a 1=a$ for all $a$.

P3. $\exists 0$, with $a+0=a$.

$\mathrm{P3}^{\prime} .0+a=a$.

P4. To each $a$ corresponds at least one $a^{\prime}$, such that $a a^{\prime}=0$ and $a+a^{\prime}=1$.

That is, multiplication is distributive on sums, we have a multiplicative rightunit, an additive zero, and right-complements. We shall now show that multiplication is idempotent, that complementation is involutory and unique, that right-complements are left-complements, that the multiplicative right-unit is also a left-unit, and that the additive zero is also a multiplicative zero. The postulates which are superfluous in each proof are listed to the right of the statement of the result.

T1. $a a=a$. (Cf. $\left[4\right.$, p. 1].) (without $\left.\mathrm{P}^{\prime}, \mathrm{P}^{\prime}\right)$.

Proof. $a=a 1=a\left(a+a^{\prime}\right)=a a+a a^{\prime}=a a+0=a a$.

T2. $\left(a^{\prime}\right)^{\prime}=a$ for all $a$ and $\left(a^{\prime}\right)^{\prime}$. (Cf. [4, p. 3].)

Proof.

$$
\begin{array}{rlr}
\left(a^{\prime}\right)^{\prime} & =0+\left(a^{\prime}\right)^{\prime}\left(a^{\prime}\right)^{\prime} & \text { by P3', T1 } \\
& =a^{\prime}\left(a^{\prime}\right)^{\prime}+\left(a^{\prime}\right)^{\prime}\left(a^{\prime}\right)^{\prime}=\left(a^{\prime}+\left(a^{\prime}\right)^{\prime}\right)\left(a^{\prime}\right)^{\prime} & \\
& =1\left(a^{\prime}\right)^{\prime}=\left(a+a^{\prime}\right)\left(a^{\prime}\right)^{\prime}=a\left(a^{\prime}\right)^{\prime}+0 & \\
& =0+a\left(a^{\prime}\right)^{\prime}=a a^{\prime}+a\left(a^{\prime}\right)^{\prime}=a\left(a^{\prime}+\left(a^{\prime}\right)^{\prime}\right) & \\
& =a \cdot 1=a . &
\end{array}
$$

Remark. Without using P2, we have shown that $\left(a^{\prime}\right)^{\prime}=a \cdot\left(a^{\prime}\right)^{\prime}=a \cdot 1$.

COROLlary 1. $a^{\prime} a=0$ and $a^{\prime} a=1$.

Corollary 2. If $a b=a c=0$ and $a+b=a+c=1$, then $b=c$; complements are unique.

For if $a^{\prime}$ is any complement of $a, b=\left(\left(a^{\prime}\right)^{\prime}\right)^{\prime}=c$.

CoROllaRY 3. $1 \cdot a=a$, for all $a$.

Proof. $1 \cdot a=\left(a+a^{\prime}\right) a=a a+a^{\prime} a=a+0=a$.

T3. $a \cdot 0=0 \cdot a=0$, for all $a$.

Proof. $0=a a^{\prime}=a\left(a^{\prime}+0\right)=a a^{\prime}+a 0=0+a 0=a 0$, and $0=a a^{\prime}=(0+a) a^{\prime}$ 
$=0 a^{\prime}+a a^{\prime}=0 a^{\prime}+0=0 a^{\prime}$, all without using P2. But by T2 every element is a complement; hence $0 a^{\prime}=0$ for all $a$ implies $0 a=0$ for all $a$.

Corollary. If $0=1$, then $0=0+0=0+a \cdot 0=0+a \cdot 1=a \cdot 1=a$; hence all elements are equal.

Remark. By T2, Corollary 1 , which is symmetric to P4, and T2, Corollary 3 , which is symmetric to $\mathrm{P} 2$, we now have complete left-right symmetry in the properties of addition and multiplication.

An easier way to guarantee this would of course be to substitute the commutative laws $a b=b a$ and $a+b=b+a$ for $\mathrm{P}^{\prime}$ and $\mathrm{P}^{\prime}$ '.

3. Even elements. We now define $1+1=2,2+2=4$, and call the leftmultiples $y 2$ of 2 even elements. This is the opposite of Newman's usage. We note that by $\mathrm{P} 1, \mathrm{P} 2$ and $\mathrm{T} 1$ alone, $4=2+2=2 \cdot 1+2 \cdot 1=2(1+1)=2 \cdot 2=2$; also, by definition, $4=(1+1)+(1+1)$.

T4. An element $x$ is even if and only if it is additively idempotent: $x+x=x$ (without $\mathrm{P}^{\prime}, \mathrm{P}^{\prime}$ ).

Proof. Clearly $y 2+y 2=y(2+2)=y 2$; conversely, if $x=x+x$, then $x=x \cdot 1$ $+x \cdot 1=x(1+1)=x 2$.

T5. Any multiple $x$ t or $u x$ of an even element $x$ is even.

Proof. If $x=x+x$, then $x t=(x+x) t=x t+x t$ and $u x=u(x+x)=u x+u x$ for all $t, u$.

T6. The correspondence $x \rightarrow x+x=x 2$ is an idempotent endomorphism; that is, $(x+y) 2=x 2+y 2,(x y) 2=(x 2)(y 2)$, and $(x 2) 2=x 2$ (without $\left.\mathrm{P}^{\prime}\right)$.

Proof. By $1^{\prime},(x+y) 2=x 2+y 2$. Again, $(x 2) 2=x 2+x 2=x(2+2)=x 2$ by $\mathrm{P} 1, \mathrm{P} 2, \mathrm{~T} 1$. Finally, by $\mathrm{P} 1, \mathrm{P} 1^{\prime}, \mathrm{P} 2, \mathrm{~T} 1$

$$
\begin{aligned}
(x 2)(y 2) & =(x+x)(y+y)=(x+x) y+(x+x) y=(x y+x y)+(x y+x y) \\
& =((x y) 2) 2=(x y) 2 .
\end{aligned}
$$

It is a corollary of $\mathrm{T} 6$ that the even elements form a subalgebra, in which addition is idempotent.

We remark that T4-T6 not only do not require $\mathrm{P}^{\prime}$, but only require P3-P4 insofar as they are needed to prove T1. That is', T4-T6 are valid in any system with idempotent multiplication and a right-unit, in which the distributive laws $\mathrm{P} 1-\mathrm{P} 1^{\prime}$ are valid. These considerations are developed further in $\$ 7$.

4. Direct decomposition theorem. Now let $2^{\prime}$ denote the right-complement of 2 , so that $2 \cdot 2^{\prime}=0,2+2^{\prime}=1$; we shall call the left-multiples of $2^{\prime}$, odd elements. Using the results of $\$ 2$, it is easy to obtain a direct decomposition theorem. 
T7. The odd elements are the additively nilpotent elements. More precisely, the conditions $x=y 2^{\prime}, x=x 2^{\prime}, x+x=x 2=0$ are equivalent.

Proof. If $x+x=0$, then $x=x\left(2+2^{\prime}\right)=x 2+x 2^{\prime}=0+x 2^{\prime}=x 2^{\prime}$. If $x=x 2^{\prime}$, then $x=y 2^{\prime}$ trivially, all without P3. Finally, $y 2^{\prime}+y 2^{\prime}=y\left(2^{\prime}+2^{\prime}\right)=y\left(2^{\prime} \cdot 2\right)$ $=y 0=0$ by $\mathrm{T} 2$, Corollary 1 , and $\mathrm{T} 3$. We remark that $\mathrm{T} 7$ follows if we have a right unit 1, a right-additive and right multiplicative zero, right distributivity, and left-complements.

T7'. Any multiple of an odd element is odd.

Proof. If $x+x=0$, then $x t+x t=(x+x) t=0 t=0$, and $u x+u x=u(x+x)$ $=u \cdot 0=0$ for all $t, u$, by $\mathrm{P} 1, \mathrm{P} 1^{\prime}, \mathrm{T} 3$.

T8. Any system satisfying $\mathrm{P} 1, \mathrm{P}^{\prime}, \mathrm{P} 2, \mathrm{P} 3, \mathrm{P}^{\prime}, \mathrm{P} 4$ is the direct union of the subalgebras of even and odd elements.

Proof. Consider the correspondences $z \rightarrow\left(z 2, z 2^{\prime}\right)=(x, y)$ and $(x, y) \rightarrow x+y$. If $x$ is even and $y$ is odd, then

$$
\begin{aligned}
& (x+y) 2=x 2+y 2=(x+x)+0=x \\
& (x+y) 2^{\prime}=x 2^{\prime}+y 2^{\prime}=(x+x) 2^{\prime}+y=y
\end{aligned}
$$

by $\mathrm{P} 1, \mathrm{~T} 7, \mathrm{P} 3$, by $\mathrm{P} 1, \mathrm{P3}^{\prime}$, since $(x+x) 2^{\prime}=x 2^{\prime}+x 2^{\prime}=x\left(2^{\prime}+2^{\prime}\right)=x 0=0$. Conversely, for any $z, z 2$ is even and $z 2^{\prime}$ odd, and $z=z \cdot 1=z\left(2+2^{\prime}\right)=z \cdot 2+z 2^{\prime}$. Hence the correspondences are one-one and reciprocal. Further, $\left(z+z_{1}\right) 2=z 2+z_{1} 2$ and $\left(z+z_{1}\right) 2^{\prime}=z 2^{\prime}+z_{1} 2^{\prime}$; hence addition is component-by-component. Finally,

$$
\begin{aligned}
(x+y)\left(x_{1}+y_{1}\right) & =\left(x x_{1}+x y_{1}\right)+\left(y x_{1}+y y_{1}\right) \\
& =\left(x x_{1}+0\right)+\left(0+y y_{1}\right) \\
& =x x_{1}+y y_{1}
\end{aligned}
$$

by $\mathrm{P} 1-\mathrm{P} 1^{\prime}$

as shown below

by $\mathrm{P} 3-\mathrm{P} 3^{\prime}$.

(To show that $x y_{1}=y x_{1}=0$, note that $x y_{1}$ and $y x_{1}$ are both even and odd by T5, T $7^{\prime}$; while if $u$ is both even and odd, then $u=u+u=0$ by definition and T7.) Hence multiplication is also component-by-component, $x x_{1}$ being even and $y y_{1}$ odd by $\mathrm{T} 5, \mathrm{~T} 7$ '.

We can now prove that the even elements form a Boolean algebra, while the odd elements form a (not necessarily associative) ring, in which multiplication is commutative and idempotent. But as we have nothing to add to Newman's proof, we shall not repeat his argument $\left({ }^{3}\right)$.

5. Postulates for distributive lattices. Instead, we shall give a new proof that the even elements form a Boolean algebra, which will yield as a byproduct a new set of postulates for distributive lattices. By confining ourselves to even elements, we have the additional postulate

$\left({ }^{3}\right)$ One can show successively $a+1=1+a, a+b=b+a, 1+(1+c)=(1+1)+c, 1+(b+c)$ $=(1+b)+c, a+(b+c)=(a+b)+c$; the trick is to right-multiply respectively by $a+a^{\prime}, b+b^{\prime}$, $c+c^{\prime}, b+b^{\prime}, a+a^{\prime}$ and expand. Cf. $[4$, p. 260]. 
P5. $a+a=a$.

Using this, we can show

T9. $a+1=1+a=1$.

Proof. By P2-T2, P1-P1', T1, T4=P5, and P3', we have

$$
\begin{aligned}
a+1 & =(a+1)\left(a+a^{\prime}\right)=(a a+1 a)+\left(a a^{\prime}+1 a^{\prime}\right) \\
& =(a+a)+\left(0+a^{\prime}\right)=a+a^{\prime}=1 .
\end{aligned}
$$

By symmetry (\$2), we get $1+a=1$.

We shall now show that any system which satisfies $\mathrm{P} 1-\mathrm{P} 1^{\prime}, \mathrm{P} 2-\mathrm{P} 2$ ', $\mathrm{T} 1$, and $\mathrm{T} 9$ is a distributive lattice with 1 . It is a corollary that the even elements in any system satisfying $\mathrm{P} 1-\mathrm{P} 1{ }^{\prime}, \mathrm{P} 2, \mathrm{P} 3-\mathrm{P} 3^{\prime}$ and $\mathrm{P} 4$ form a Boolean algebra (complemented distributive lattice). We shall prove the usual postulates for a distributive lattice as a chain of identities.

T10. $a+a=a$.

Proof. By P2, T9, P1, T1, and with P5, we have $a=a 1=a(a+1)=a a+a \cdot 1$ $=a+a$.

T11. $a b+a=a+a b=a+b a=b a+a=a$.

Proof. $a+a \cdot 1=a(b+1)=a b+a 1=a b+a$. The other proofs are entirely similar.

T12. $a(a+b)=a(b+a)=(a+b) a=(b+a) a=a$.

Proof. $a(a+b)=a a+a b=a+a b=a$, by $\mathrm{P} 1, \mathrm{~T} 1, \mathrm{~T} 11$. The other proofs are entirely similar.

T13. $a+b=b+a$.

Proof. By T12, P1, P1', T12, we have

$$
\begin{aligned}
a+b & =a(b+a)+b(b+a)=(a+b)(b+a) \\
& =(a+b) b+(a+b) a=b+a .
\end{aligned}
$$

T14. $a[(a+b)+c]=a, b[(a+b)+c]=b, c[(a+b)+c]=c$.

Proof. By P1, T12, and T1, we have

$$
\begin{aligned}
& a[(a+b)+c]=a(a+b)+a c=a+a c=a, \\
& b[(a+b)+c]=b(a+b)+b c=b+b c=b, \\
& c[(a+b)+c]=c(a+b)+c c=c(a+b)+c=c .
\end{aligned}
$$

T15. $a+(b+c)=(a+b)+c$.

Proof. $a+(b+c)=a[(a+b)+c]+(b[(a+b)+c]+c[(a+b)+c])$ by T14. 
By $\mathrm{P} 1$ ', this is $[a+(b+c)][(a+b)+c]$. By left-right symmetry, this can be shown to be $(a+b)+c$.

T16. $a+b c=(a+b)(a+c)$ and $a b+c=(a+c)(b+c)$.

Proof. By P1-P1', T1, T12, T15, and T11, we have

$$
\begin{aligned}
(a+b)(a+c) & =a(a+c)+b(a+c)=a+(b a+b c) \\
& =(a+b a)+b c=a+b c .
\end{aligned}
$$

The other identity follows by symmetry.

We now define the dual of an identity to be the equality obtained from it by interchanging addition and multiplication. Thus $\mathrm{P} 1-\mathrm{P} 1^{\prime}$ and $\mathrm{T} 16$ are dual; T1 and T10 are dual; T11 and T12 are dual. Since the proofs of T13 and T15 involve only these laws, it follows that dual proofs can be made to show

T17. $a b=b a$.

T18. $a(b c)=(a b) c$.

But $\mathrm{T} 1, \mathrm{~T} 10, \mathrm{~T} 17, \mathrm{~T} 13, \mathrm{~T} 18, \mathrm{~T} 15, \mathrm{~T} 12, \mathrm{~T} 13$, and $\mathrm{P} 1-\mathrm{P} 1{ }^{\prime}$ are the usual postulates for a distributive lattice; this completes our demonstration.

Remark. 1. Since the postulates for a distributive lattice are self-dual, it follows that $\mathrm{T} 16, \mathrm{P} 3-\mathrm{P}^{\prime}, a 0=0 a=0$, and $\mathrm{T} 10$ are postulates for a distributive lattice with zero.

Remark 2. Just after T12, we can easily show that the conditions $a+b=a$, $b+a=a, a b=b$, and $b a=b$ are all equivalent to each other-and hence define $a \geqq b$ in a self-dual manner. We can show $a \geqq a$, that $a \geqq b$ and $b \geqq a$ imply $a=b$. But we need T15 or T18 to prove transitivity.

6. Subdirect decomposition theorem. We shall now use an entirely different and shorter argument to characterize the most general direct union of a Boolean algebra and an associative Boolean ring.

TheOREM. The most general algebra satisfying P1-P1', P2-P2', P3, P4, and P6, $(a b) c=(a c)(b c)$, is a direct union of a Boolean algebra and an associative Boolean ring.

Proof. Each identity is valid in every homomorphic image of an algebra $A$, if it is valid in $A$. It is also valid in the direct union $A \oplus B$, if it is valid in $A$ and $B$ individually. Hence it is valid in any subdirect union (in the sense of [1]) of algebras in which it holds. It follows, by the principal conclusion of [1], that all consequences of these identities which hold in every subdirectly irreducible algebra satisf ying them, hold in every algebra satisfying them.

But now observe that (by definition) every correspondence $x \rightarrow x a$ of an algebra $A$ is an endomorphism, if and only if $\mathrm{P}^{\prime}$, $\mathrm{P} 6$ hold.

LEMMA 1. Any algebra which satisfies P1-P1', P2-P2', P3, P4, P6, and contains an element a not 0 or 1 , is subdirectly reducible. 
Proof. Since the correspondences $x \rightarrow x a, x \rightarrow x a^{\prime}$ are endomorphisms ( $\mathrm{P} 1^{\prime}$, P6), the correspondence $x \rightarrow\left(x a, x a^{\prime}\right)$ is a homomorphism of $A$ onto a subdirect union. Since $x a=y a$ and $x a^{\prime}=y a^{\prime}$ imply (P2, P4, P1)

$$
x=x 1=x\left(a+a^{\prime}\right)=x a+x a^{\prime}=y a+y a^{\prime}=y\left(a+a^{\prime}\right)=y 1=y,
$$

this homomorphism is an isomorphism. Again, by T1, $a a=a$ and by $\mathrm{P} 2$ ', $1 a=a(a \neq 1)$, while $a^{\prime} a^{\prime}=a^{\prime}$ and $1 a^{\prime}=a^{\prime}$ similarly, where $a^{\prime}=1$ would imply $0=a a^{\prime}=a 1=a$, contrary to hypothesis. Hence both endomorphisms determine proper congruence relations, and $A$ is subdirectly reducible.

LemMA 2. The only algebras consisting of 0 and 1 which satisfy $\mathrm{P} 1-\mathrm{P} 1^{\prime}$, P2-P2', P3, P4, P6 are the Boolean algebra of two elements and the Boolean ring of two elements.

Proof. By P2, P2', P3, we have

$$
0 \cdot 1=1 \cdot 0=0, \quad 1 \cdot 1=1, \quad 0+0=0, \quad 1+0=1 .
$$

Since $0+0=0,0^{\prime}$ cannot be 0 and must be 1 . This gives, by $\mathrm{P} 4$,

$$
0+1=1 \text {. }
$$

Again, $0 \cdot 0=1$ would imply the contradiction

$$
1=0 \cdot 0=(1 \cdot 0) \cdot 0=(1 \cdot 0) \cdot(0 \cdot 0) \text { (by P6) }=0 \cdot 1=0 ;
$$

hence $0 \cdot 0=0$. The only sum or product not determined is $1+1$; the possibilities $1+1=1$ and $1+1=0$ give the two cases mentioned in Lemma 2 .

Corollary. Any algebra satisfying P1-P1', P2-P2', P3, P4, and P6 is a subdirect union of Boolean algebras and Boolean rings of two elements.

It is a further corollary that addition and multiplication are commutative and associative, and indeed that all the results of $\$ \S 2-4$ hold.

Remark 1 . We can replace $\mathrm{P}^{\prime}$ by $0 a=0$ in the preceding argument: in the proof of Lemma 1, $0 a^{\prime}=a a^{\prime}=0$, where $0 \neq a$, and $\left(a^{\prime}\right)\left(a^{\prime}\right)^{\prime}=0=0\left(a^{\prime}\right)^{\prime}$, where $a^{\prime}=0$ would imply $a=a+a^{\prime}=a+0=1$, hence $x \rightarrow\left(x a^{\prime}, x\left(a^{\prime}\right)^{\prime}\right)$ subdirectly reduces $A$. In Lemma $2,1 \cdot 0=0$ is lost in (1); but since $1 \cdot 1=1,1^{\prime} \neq 1$, hence $1^{\prime}=0$ and $1 \cdot 0=1 \cdot 1^{\prime}=0$ still holds.

Remark 2. Thus replacing $\mathrm{P}^{\prime}$ in $\$ 2$ by $\mathrm{P} 6$ and $\mathrm{P} 2^{\prime}$ or $0 a=a$ effectively guarantees that the odd elements form an associative ring; otherwise it has no effect.

Remark 3. We can prove analogous results for systems satisfying P1, P2, P3', T9, P6, $a b+c=(a+c)(b+c),(a+c)+(b+c)=(a+b)+c$ and the commutative law of multiplication. By assumption, the correspondences $x \rightarrow x a$ and $x \rightarrow x+a$ are endomorphisms, for all $a$. Again, $a=(1 \cdot 1) a=(1 a)(1 a)=a a$. It follows that if $x a=y a$ and if $x+a=y+a$, then 


$$
\begin{aligned}
x=x 1 & =x(1+a)=x+x a=x x+x a=x(x+a)=x(y+a) \\
& =x y+x a=y x+y a=y(x+a)=y(y+a)=y .
\end{aligned}
$$

Thus the correspondence $x \rightarrow(x a, x+a)$ is a isomorphism. Again $1 a=a a$ and $0+a=a=a(1+1)=a+a$; hence any system containing an $a \neq 0,1$ is subdirectly reducible. And the only system of 0 and 1 satisfying our postulates is a distributive lattice.

7. Self-dual and symmetric postulates; counterexamples. We have already observed that the laws of Boolean algebra are left-right symmetric for addition and multiplication ( $\$ 2$, Remark), and self-dual under interchange of addition and multiplication ( $\$ 5$, Remark 1$)$. Thus they are invariant under an octic group of symmetries $\left({ }^{4}\right)$. The same remark applies to distributive lattices.

This suggests introducing the group of symmetries on the postulates as a "metamathematical" postulate, and seeing how few other postulates are required. It is evident that the following three are sufficient for Boolean algebra:

P1. $a(b+c)=a b+a c . \quad$ P2. $a 1=a . \quad$ P4. $a+a^{\prime}=1$.

For we get $\mathrm{P} 1, \mathrm{P}^{\prime}, \mathrm{P} 2, \mathrm{P} 3, \mathrm{P} 3^{\prime}, \mathrm{P} 4$ immediately, and from $\mathrm{T} 1$, by dualization, we get $a+a=a$.

This easy success suggests trying to see whether P1, P2, and their transforms under the octic group of left-right symmetries and duality do not constitute a sufficient set of postulates for a distributive lattice. In fact, one can prove directly that

$$
\begin{aligned}
1=0+1 & =(0 \cdot 1)+1=(0+1)(1+1)=1 \cdot(1+1) \\
& =1 \cdot 1+1 \cdot 1=1+1,
\end{aligned}
$$

by the dual-symmetric $\mathrm{P}^{\prime}$ of $\mathrm{P} 2, \mathrm{P} 2$, the dual-symmetric of $\mathrm{P} 1, \mathrm{P} 3^{\prime}$ again, $\mathrm{P} 1$, and $\mathrm{P} 2$ respectively. Multiplying through by $a$ (using $\mathrm{P} 2$ ), and dualizing, we get idempotence:

$$
a+a=a, \quad a a=a .
$$

Furthermore, consider the "free algebra" generated by $0,1, a$. Let $s, s_{1}, s_{2}, \ldots$ denote generically sums of terms 1 and $a$, and $p, p_{1}, p_{2}, \cdots$ denote dually products of terms 0 and $a$. We can prove by induction that all elements other than $0,1, a$ are such sums or products.

Indeed, from the cases $s=1$ and $s=a$, it follows by induction since $a\left(s+s_{1}\right)=a s+a s_{1}$ and $a+a=a$ that

$$
a s=s a=a, \text { whence } a+p=p+a=a,
$$

(4) This is in accordance with the philosophic principle that "The final form of any scientific theory $T$ is (1) based on a few simple postulates, and (2) contains an extensive ambiguity, associated symmetry, and underlying group $G$, in such wise that . . T appears nearly selfevident in view of the Principle of Sufficient Reason." (G. D. Birkhoff) 
by duality. We shall now prove that

$$
s p=a p=p_{1}
$$

Indeed, $a p=a p,(s+1) p=s p+p=a p+p p$ (by induction and (1)) $=(a+p) p$ $=a p$ by (2), and $\left(s+s^{\prime}\right) p=s p+s^{\prime} p=a p+a p$ (by induction) $=a p$ by (1). This completes the proof of (3). By duality, we get

$$
s+p=s+a=s_{1}
$$

Since $s+s=s s=s$ and $p+p=p p=p$, we have a homomorphism of the "free algebra" onto the system with five elements $0,1, a, s, p$ and the rules of operation described by P1, P2, (1)-(4) and their left-right symmetric and dual transforms. This self-dual and symmetric image algebra however satisfies P1, P2; hence P1, P2 and their transforms do not constitute a set of postulates for distributive lattices. (Though by $\S 5, \mathrm{P} 1, \mathrm{P} 2$, and T9 and their transforms do.)

An even more simple counterexample consists of two elements: $0=1$ and $a$, with identical addition and multiplication tables given by:

$$
\begin{aligned}
& 00=0+0=0, \\
& 0 a=a 0=0+a=a+0=a a=a+a=a .
\end{aligned}
$$

In this system, addition and multiplication are idempotent, commutative, and associative; all distributive laws hold; 0 is an additive and 1 a multiplicative unit; $a$ is a multiplicative zero and additive unity. Moreover by forming the direct product of it and the Boolean algebra of two elements, we can make $0 \neq 1$.

It would be interesting to determine what were the different elements of the form $s$ and $p$ in the "free" self-dual and symmetric algebra generated by $a$ (with 0 and 1 ) subject to P1 and P2.

It would also be interesting to determine the independent subsets of the postulates (for distributive lattices) generated by $\mathrm{P} 1, \mathrm{P} 2, \mathrm{~T} 9$ and their transforms under the octic group of left-right symmetries and duality.

\section{BIBLIOGRAPHY}

1. G. Birkhoff, Subdirect unions in universal algebra, Bull. Amer. Math. Soc. vol. 50 (1944) pp. 764-768.

2. George Boole, The laws of thought, 1854, republished by the Open Court Publishing Co.

3. E. V. Huntington, New sets of independent postulates for the algebra of logic, Trans. Amer. Math. Soc. vol. 35 (1933) pp. 274-304; corrections on pp. 557, 971.

4. M. H. A. Newman, A characterization of Boolean algebras and rings, J. London Math. Soc. vol. 16 (1941) pp. 256-272.

5. M. H. Stone, Representations of Boolean algebras, Trans. Amer. Math. Soc. vol. 40 (1936) pp. 37-111.

HARVARD UNIVERSITY,

Cambridge, Mass. 